\title{
CONDITIONS OF ACCELERATION OF SOLAR ELECTRONS, AND DETERMINATION OF THE MAGNETIC FIELD IN THE HIGH CORONA FROM THE CHARACTERISTICS OF A TYPE-IV BURST
}

\author{
A. Boischot and B. Clavelier \\ (Observatoire de Paris-Meudon, France)
}

\begin{abstract}
A BSTRACT
The study of a moving type-IV burst shows that acceleration of electrons can happen in the high corona, and a mechanism is proposed for such an acceleration. The low-frequency cut-off of radio spectrum is interpreted as due to the effect of the coronal plasma upon the synchrotron emission, and this leads to an accurate determination of the intensity of the magnetic field at an altitude of $1 R_{\odot}$. Then it is possible to compute the energy and the density of the relativistic electrons.
\end{abstract}

On September 14, 1966, a flare was observed on the West limb of the Sun, more precisely on an active center located $10^{\circ}$ behind the limb. For this event several types of solar radio bursts were detected, mainly a type II beginning at $10 \cdot 17 \mathrm{UT}$, a first type IV at $10.43 \mathrm{UT}$, and a second one at $11.00 \mathrm{UT}$. The latter has been studied at Nançay on 408 and $169 \mathrm{MHz}$ and may be described as follows:

(1) The emission begins at exactly the same time on both frequencies, and has the same profile (Figure 1). This is also true for all other frequencies on which the burst has been observed.

(2) The position of the centres of emission determined on 169 and $408 \mathrm{MHz}$ shows three remarkable characteristics (Figure 2):

(a) The emissions on 169 and $408 \mathrm{MHz}$ come from the same source;

(b) When the burst starts, this source is already located at an altitude of one solar radius (this is a minimum value, assuming a radial position relative to the optical flare.

(c) The source moves upward with a constant velocity of $530 \mathrm{~km} / \mathrm{s}$ and was followed up to an altitude of two solar radii (in the radial hypothesis).

(3) The size of the source has the same value on 169 and $408 \mathrm{MHz}$ and is constant during the main part of the burst. This source size, $80000 \pm 10000 \mathrm{~km}$ is much smaller than the displacement of the source during its ascension through the corona. Only near the end of the burst the source increases rapidly in size.

These characteristics are those of a moving metric type IV (Kundu, 1965). It is generally accepted that this type of burst is due to synchrotron emission by relativistic

Kiepenheuer (ed.), Structure and Development of Solar Active Regions, 565-569. (C. I.A.U. 


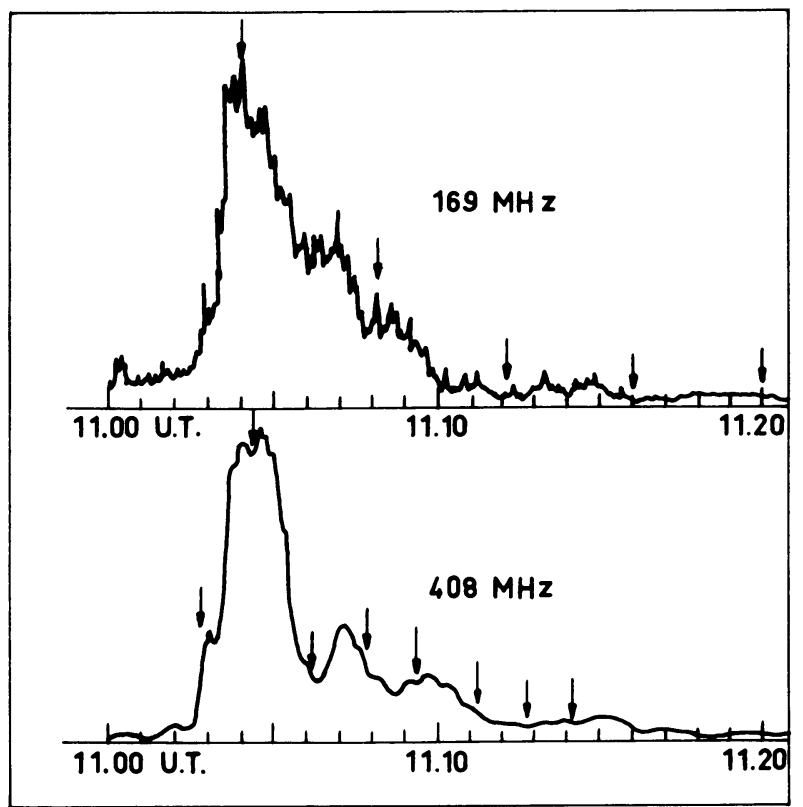

FIG. 1. The second type IV of the September 14 event, observed on $169 \mathrm{MHz}$ and $408 \mathrm{MHz}$. The arrows show the times at which the position of the centre of emission has been determined.

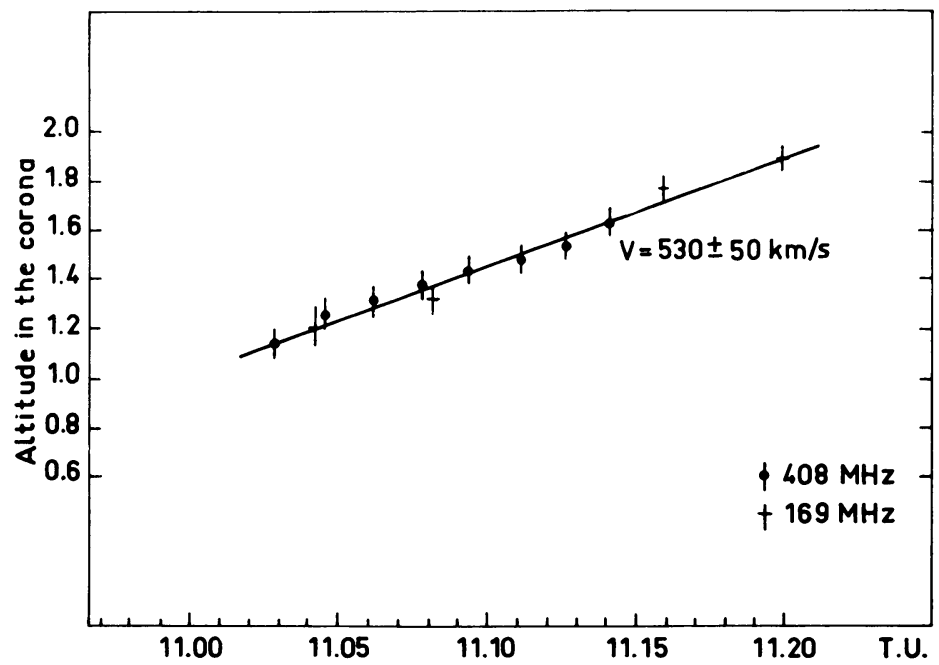

Fig. 2. Position of the centre of emission on $169 \mathrm{MHz}$ and $408 \mathrm{MHz}$. Altitudes and velocity are given assuming a radial outward motion. 
electrons accelerated during the flare. But up to now it was difficult to study the properties of these electrons because of the lack of information about the source of the emission. The present observations allow us to make a finer analysis of this problem.

\section{Mechanism of Acceleration of the Electrons}

When the burst starts, the source is already at an altitude of $700000 \mathrm{~km}$ in the corona. This is much higher than the critical heights where the plasma frequency is equal either to $169 \mathrm{MHz}$ or $408 \mathrm{MHz}$. Then we are sure that the waves suffer no perturbation, cut-off or refraction, from the coronal plasma and that we are observing the source exactly as it is. The fact that the emission starts at exactly the same time on each frequency supports this conclusion, for any effect of the plasma would be chromatic.

As the emission begins only at $1 R_{\odot}$, we have to conclude that the relativistic electrons do not exist lower, or in other words that the acceleration takes place at this altitude, where nothing noticeable is seen in the visible range. Moreover, the burst shows a rapid increase $(1 \mathrm{~min})$ followed by a much longer quasi-exponential decrease, and this means that the acceleration takes place in a relatively narrow layer of $60000 \mathrm{~km}$ at the most.

Mangeney and Lacombe proposed a mechanism to explain this acceleration by the action of perturbation coming from the chromosphere and ascending in the corona with the measured speed of $530 \mathrm{~km} / \mathrm{s}$. (Boischot et al., 1967).

When the Alfvén-Mach number is greater than 1, the perturbation propagates as a soliton, and an instability occurs for a critical Mach number equal to

$$
M^{*}=1+{ }_{8}^{3}\left(\begin{array}{c}
8 \pi N_{\mathrm{e}} k T \\
H^{2}
\end{array}\right) .
$$

This instability leads to plasma oscillations and turbulence in which the electrons are accelerated by the mechanism described by Tsytovich. The acceleration then takes place at the altitude where the perturbation attains this critical Mach number.

For a velocity of $500 \mathrm{~km} / \mathrm{s}$, and $T=2 \times 10^{6}{ }^{\circ} \mathrm{K}$, this happens when $N_{\mathrm{e}}=10^{7} \mathrm{~cm}^{-3}$ and $H=0.5$ gauss, i.e., at an altitude of $1 R_{\odot}$ as we shall determine later on.

\section{Influence of the Coronal Plasma upon the Synchrotron Radiation (Razin effect)}

The burst spectrum is given in Figure 3. It shows sharp cut-off toward high and low frequencies, and cannot be interpreted by any energy spectrum of the electrons in classical synchrotron theory.

The low-frequency cut-off has a slope at about +4 , and cannot be explained either by a low-energy cut-off in the energy spectrum, or by free-free or synchrotron absorption. 


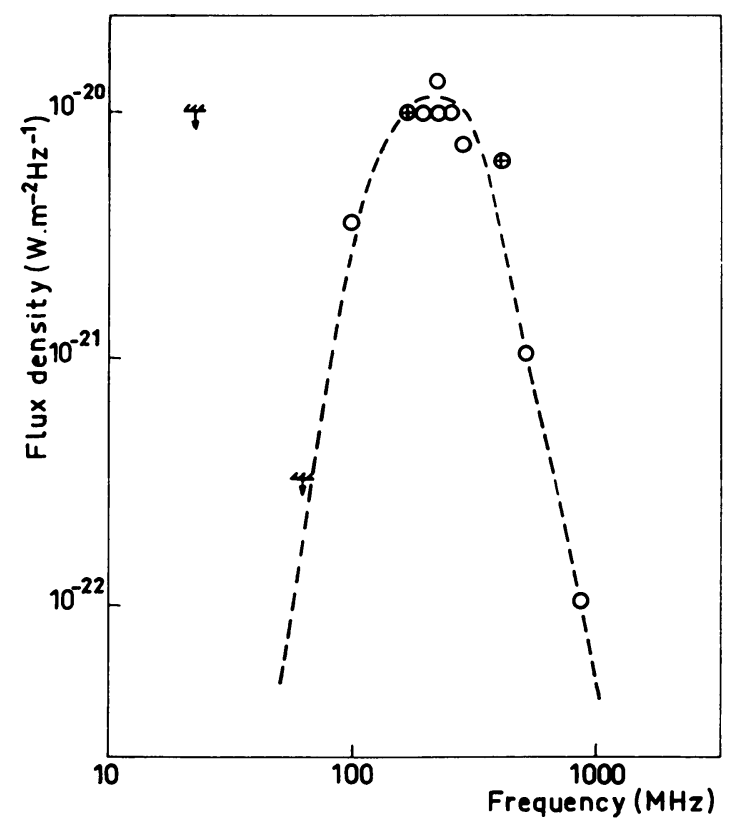

FIG. 3. Spectrum of the moving type IV of September 14, 1966.

This low-frequency cut-off is due to the effect of the coronal plasma on the synchrotron emission. The synchrotron effect is generally computed by assuming that the electrons radiate in free space, with a refractive index equal to unity. But it is known that if we take account of a departure of $n$ from unity, the emission on low frequencies is much less intense, giving a quasi-exponential cut-off (Ginzburg and Syrovatskii, 1965).

It is shown that this effect is noticeable at frequencies smaller than a critical frequency given by

$$
v_{c}=20 \stackrel{N_{\mathrm{e}}}{H},
$$

where $v_{c}$ is in Hertz, $N_{\mathrm{e}}$ the density of the thermal electrons in $\mathrm{cm}^{-3}$ and $H$ the magnetic field in gauss.

In our case, $v_{c}=400 \mathrm{MHz}$, and we can take $N_{\mathrm{e}}=10^{7} \mathrm{~cm}^{-3}$ at an altitude of $1 R_{\odot}$. This leads to $H=0.5$ gauss and gives one of the more accurate determinations of the magnetic field in the high corona.

\section{Energy and Density of the Relativistic Electrons}

The high-frequency part of the radio spectrum has a very steep slope, of spectral index -4 if we assume a power law. This would correspond to an energy spectrum 
of the electrons of the same shape with a spectral index of +9 . But this high-frequency slope is more likely due to a high-energy cut-off of the electrons.

On the other side, the effect of the coronal plasma upon the synchrotron mechanism prevents a good determination of the low-energy part of the electron spectrum because the corresponding electrons radiate very little.

Then, in a first approximation, it is possible to explain the observed spectrum by quasi-monoenergetic electrons, and, in this case, to compute their energy and density.

The maximum of the spectrum may be estimated, in absence of Razin effect, to be around $25 \mathrm{MHz}$, and the maximum intensity $10^{-20} \mathrm{~W} \cdot \mathrm{m}^{-2} \mathrm{~Hz}^{-1}$.

From the frequency of maximum and the value of magnetic field which has been determined above, it is possible to derive the energy of the electrons by the classical formulae:

$$
v_{m}=4.6 \times 10^{-6} H_{\perp} E_{e v}^{2} .
$$

We find an energy of $3 \mathrm{MeV}$. The density of the relativistic electrons is then given by

$$
N=7 \cdot 2 \times 10^{22} \frac{I_{v}}{L H}
$$

where $I_{v}$ is the brightness of the source and $L$ its linear dimension.

Finally, assuming that the source has in the three dimensions the same size that has been measured radially, we find for the density of the relativistic electrons: $N=6000 \mathrm{el} / \mathrm{cm}^{3}$.

\section{References}

Boischot, A., Clavelier, B. (1967) Astrophys. Lett., 1, 7.

Boischot, A., Clavelier, B., Lacombe, C., Mangeney, A. (1967) $\quad$ C.R. Acad. Sci., 265, 1151.

Ginzburg, V.L., Syrovatskii, S. I. (1965) A. Rev. Astr. Astrophys., 3, 297.

Kundu, M.R. (1965) Solar Radio Astronomy, Interscience Publishers, New York, p. 383.

\section{DISCUSSION}

Krüger: In principle, spectra with a small frequency extent could be expected also when there are particles of a very limited energy range, but this can be excluded in your case?

Boischot: In the classical theory of synchrotron emission it is shown that if there is a low-energy cut-off in the spectrum of electrons, or even in the case of monoenergetic electrons, the spectral index of the low-energy part of the radio emission cannot be larger than $1 / 3$. This leads to much wider spectrum than observed.

Elske Smith: I think you have to be cautious in using the electron density in the corona in your relation to find the magnetic field. If there is an inhomogeneity in the corona because of the association with an active region, there may be an increase in the electron density by a factor 3 , perhaps even 10, leading to a corresponding decrease in the magnetic field.

Boischot: I think that the coronal density over an active centre is known within a factor of 2 or 3 . Now, the main inaccuracy comes probably from the determination of $v_{\mathrm{c}}$ from the observed spectrum, but more detailed calculations for different energy spectra of electrons must improve this point. 